Odd asymmetric factorization of Wiener-Hopf plus Hankel operators on variable exponent Lebesgue spaces

L. P. Castro and A. S. Silva

Citation: 1798, 020038 (2017); doi: 10.1063/1.4972630

View online: http://dx.doi.org/10.1063/1.4972630

View Table of Contents: http://aip.scitation.org/toc/apc/1798/1

Published by the American Institute of Physics 


\title{
Odd Asymmetric Factorization of Wiener-Hopf Plus Hankel Operators on Variable Exponent Lebesgue Spaces
}

\author{
L. P. Castro ${ }^{1, a), b)}$ and A. S. Silva ${ }^{1, c)}$ \\ ${ }^{1}$ CIDMA - Center for Research and Development in Mathematics and Applications, Department of Mathematics, \\ University of Aveiro, 3810-193 Aveiro, Portugal. \\ a)Corresponding author: castro@ua.pt \\ b)URL: http://sweet.ua.pt/castro/ \\ c)anabela.silva@ua.pt
}

\begin{abstract}
The main goal of this paper is to obtain an invertibility criterion for Wiener-Hopf plus Hankel operators acting between variable exponent Lebesgue spaces on the real line. This is obtained by a so-called odd asymmetric factorization which is applied to the Fourier symbols of the operators under study.
\end{abstract}

\section{INTRODUCTION}

The study of Wiener-Hopf and Hankel operators has a long history and is being motivated by both theoretic and applied considerations. From the theoretical point of view, the motivations to study such operators are also associated with several other classes of operators, like the Toeplitz and singular integral operators, and within the scope of linear operator theory and mathematical analysis. In particular, it is recognized a strong theoretical interest in obtaining characterizations of the boundedness and invertibility properties of such classes of operators. From a more applied perspective, that operators are being useful in a great variety of situations where they take a crucial role, e.g. by helping on the formulation of the original applied problem into an operator theory language. This is the case e.g. in wave diffraction theory $[7,8]$ where Wiener-Hopf plus Hankel operators $[2,3,4,9,10,11]$ are characterizing the equations (or systems of equations) which model some of that problems. Namely, this occurs when the geometry of diffraction objects present certain types of symmetry which give rise to the sum of a Wiener-Hopf and a Hankel operator. In view of this, the so-called regularity properties [6] of those operators are important to be known.

In the present paper we are concerned with Wiener-Hopf plus Hankel operators in a variable exponent Lebesgue spaces $[15,20,21,22,22]$ setting. The main aim is to characterize the invertibility of these Wiener-Hopf plus Hankel operators through a factorization $[12,14,17]$ of the Fourier symbols of the operators. In view of this, we will propose a certain factorization which will reveal to be adequate to that goal.

We will now introduce some notations which will be necessary for the implementation of the consequent results.

Let $p: \mathbb{R} \rightarrow[1, \infty]$ be a measurable a.e. finite function. We denote by $L^{p(\cdot)}(\mathbb{R})$ the set of all complex-valued functions $f$ on $\mathbb{R}$ such that

$$
I_{p(\cdot)}\left(\frac{f}{\lambda}\right):=\int_{\mathbb{R}}\left|\frac{f(x)}{\lambda}\right|^{p(x)} d x<\infty
$$

for some $\lambda>0$. This set becomes a Banach space when endowed with the norm

$$
\|f\|_{p(\cdot)}:=\inf \left\{\lambda>0: I_{p(\cdot)}(f / \lambda) \leq 1\right\} .
$$

The space $L^{p(\cdot)}(\mathbb{R})$ is referred as the variable exponent Lebesgue space, and if $p(\cdot)=p$ is constant, then $L^{p(\cdot)}(\mathbb{R})$ is nothing but the standard Lebesgue space $L^{p}(\mathbb{R})$.

We will suppose that

$$
1<p_{-}:=\operatorname{ess}_{x \in \mathbb{R}} \inf p(x) \leq \operatorname{ess}_{x \in \mathbb{R}} \sup p(x)=: p_{+}<\infty .
$$


Under these conditions, the space $L^{p(\cdot)}(\mathbb{R})$ is separable and reflexive, and its dual space is isomorphic to $L^{q(\cdot)}(\mathbb{R})$, where $q(\cdot)$ is the conjugate exponent function of $p(\cdot)$, defined by

$$
\frac{1}{p(x)}+\frac{1}{q(x)}=1, \quad x \in \mathbb{R} .
$$

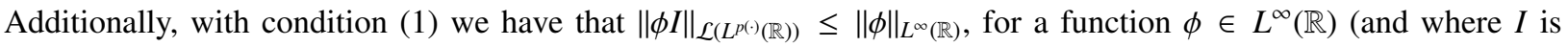
denoting the identity operator).

Moreover, $L^{p(\cdot)}\left(\mathbb{R}_{+}\right)$denotes the variable exponent Lebesgue space of complex-valued functions on the positive half-line $\mathbb{R}_{+}:=(0,+\infty)$. The subspace of $L^{p(\cdot)}(\mathbb{R})$ formed by all functions supported in the closure of $\mathbb{R}_{+}$is denoted by $L_{+}^{p(\cdot)}(\mathbb{R})$ and $L_{-}^{p(\cdot)}(\mathbb{R})$ represents the subspace of $L^{p(\cdot)}(\mathbb{R})$ formed by all the functions supported in the closure of $\mathbb{R}_{-}:=(-\infty, 0)$.

We are now in condition to identify in a mathematical way the main object of this work. We will consider Wiener-Hopf plus Hankel operators acting between Lebesgue spaces with variable exponent $p(\cdot)$, denoted by

$$
W_{\phi}+H_{\phi}: L_{+}^{p(\cdot)}(\mathbb{R}) \rightarrow L^{p(\cdot)}\left(\mathbb{R}_{+}\right),
$$

with $W_{\phi}$ and $H_{\phi}$ being Wiener-Hopf and Hankel operators defined by

$$
W_{\phi}=r_{+} \mathcal{F}^{-1} \phi \cdot \mathcal{F}, \quad H_{\phi}=r_{+} \mathcal{F}^{-1} \phi \cdot \mathcal{F} J,
$$

respectively. Here, $r_{+}$represents the operator of restriction from $L^{p(\cdot)}(\mathbb{R})$ onto $L^{p(\cdot)}\left(\mathbb{R}_{+}\right), \mathcal{F}^{-1}$ denotes the inverse of the Fourier transformation $\mathcal{F}, \phi$ is the so-called Fourier symbol, and $J: L_{+}^{p(\cdot)}(\mathbb{R}) \rightarrow L^{p(\cdot)}(\mathbb{R})$ is the reflection operator given by the rule

$$
J \varphi(x):=\widetilde{\varphi}(x):=\varphi(-x)
$$

which throughout the paper will be always considered for even functions $p(\cdot)$ (so that $J$ will therefore be a bounded operator in those variable exponent Lebesgue spaces).

In what follows, we will use the notation $\mathcal{G} B$ for the group of all invertible elements of a Banach algebra $B$.

\section{AUXILIARY RESULTS}

The boundedness of a wide variety of operators (and in particular of Wiener-Hopf and Hankel operators) follows from the boundedness of the maximal operator on variable exponent Lebesgue spaces.

Given $f \in L_{\text {loc }}^{1}(\mathbb{R})$, the Hardy-Littlewood maximal operator $M$ is defined by

$$
(M f)(x):=\sup _{x \in \Omega} \frac{1}{|\Omega|} \int_{\Omega}|f(y)| d y,
$$

where the supremum is taken over all intervals $\Omega \subset \mathbb{R}$ containing $x$, and the Cauchy singular integral operator $S$ is defined by

$$
(S f)(x):=\frac{1}{\pi i} \int_{\mathbb{R}} \frac{f(\tau)}{\tau-x} d \tau,
$$

where the integral is understood in the principal value sense.

Theorem 1 (cf. e.g. [19, Theorem 2.1.]) Let $p: \mathbb{R} \rightarrow[1, \infty]$ be a measurable function satisfying (1). If the HardyLittlewood maximal operator $M$ is bounded on $L^{p(\cdot)}(\mathbb{R})$, then the Cauchy singular integral operator $S$ is bounded on $L^{p(\cdot)}(\mathbb{R})$.

The following result states a sufficient condition on $p(\cdot)$ for $M$ to be bounded on $L^{p(\cdot)}(\mathbb{R})$.

Theorem 2 (cf. e.g. $[15,16]$ ) Let $p: \mathbb{R} \rightarrow[1, \infty]$ satisfy (1). In addition, suppose that there exist constants $A_{0}$ and $A_{\infty}$ such that $p(\cdot)$ satisfies

$$
|p(x)-p(y)| \leq \frac{A_{0}}{-\log |x-y|}, \quad|x-y| \leq \frac{1}{2},
$$


and

$$
|p(x)-p(y)| \leq \frac{A_{\infty}}{\log (e+|x|)}, \quad|x| \leq|y| .
$$

Then, the Hardy-Littlewood maximal operator is bounded on $L^{p(\cdot)}(\mathbb{R})$.

Let $\mathcal{P}(\mathbb{R})$ denote the class of exponents $p: \mathbb{R} \rightarrow[1, \infty]$ continuous on $\mathbb{R}$ satisfying $(1)$, and let $\mathcal{B}(\mathbb{R})$ denote the set of all $p(\cdot) \in \mathcal{P}(\mathbb{R})$ such that $M$ is bounded on $L^{p(\cdot)}(\mathbb{R})$. Additionally, $\mathcal{B}_{e}(\mathbb{R})$ will represent the set of all even functions $p(\cdot)$ in $\mathcal{B}(\mathbb{R})$.

A function $\phi \in L^{\infty}(\mathbb{R})$ is called a Fourier multiplier on $L^{p(\cdot)}(\mathbb{R})(p(\cdot) \in \mathcal{B}(\mathbb{R}))$ if the convolution operator

$$
W_{\phi}^{0}:=\mathcal{F}^{-1} \phi \cdot \mathcal{F}
$$

acting on $L^{2}(\mathbb{R}) \cap L^{p(\cdot)}(\mathbb{R})$, extends uniquely to a bounded operator on $L^{p(\cdot)}(\mathbb{R})$. The set of all Fourier multipliers on $L^{p(\cdot)}(\mathbb{R})$ is denoted by $\mathcal{M}_{p(\cdot)}(\mathbb{R})$.

It follows that for $\phi \in \mathcal{M}_{p(\cdot)}(\mathbb{R})$ with $p(\cdot) \in \mathcal{B}_{e}(\mathbb{R})$, the Wiener-Hopf and the Hankel operators defined in (2), $W_{\phi}: L_{+}^{p(\cdot)}(\mathbb{R}) \rightarrow L^{p(\cdot)}\left(\mathbb{R}_{+}\right)$and $H_{\phi}: L_{+}^{p(\cdot)}(\mathbb{R}) \rightarrow L^{p(\cdot)}\left(\mathbb{R}_{+}\right)$, are bounded. These are in fact necessary and sufficient conditions for the Wiener-Hopf plus Hankel operator to be bounded in variable exponent Lebesgue spaces.

Let $\ell_{0}: L^{p(\cdot)}\left(\mathbb{R}_{+}\right) \rightarrow L_{+}^{p(\cdot)}(\mathbb{R})$ denote the zero extension operator from the space $L^{p(\cdot)}\left(\mathbb{R}_{+}\right)$onto the space $L_{+}^{p(\cdot)}(\mathbb{R})$. Consider the projection operator

$$
P:=\mathcal{F} \ell_{0} r_{+} \mathcal{F}^{-1}
$$

and its complementary projection

$$
Q:=I-P .
$$

Indeed, if $p(\cdot) \in \mathcal{B}(\mathbb{R})$, then $P, Q \in \mathcal{L}\left(L^{p(\cdot)}(\mathbb{R})\right)$ and we have $P^{2}=P$ and $Q^{2}=Q$ (cf. [19, Lemma 3.10]). Additionally, from [19, Lemma 3.11], $S^{*}=S, P^{*}=P$ and $Q^{*}=Q$ are bounded linear operators in $L^{q(\cdot)}(\mathbb{R})$.

By analogy with [5, Proposition 2.10], we derive the following relations between Wiener-Hopf and Hankel operators acting on variable exponent Lebesgue spaces.

Proposition $3 \quad$ Let $\phi, \varphi \in \mathcal{M}_{p(\cdot)}(\mathbb{R})$ with $p(\cdot) \in \mathcal{B}_{e}(\mathbb{R})$ and consider

$$
W_{\phi}, W_{\varphi}, H_{\phi}, H_{\varphi} \in \mathcal{L}\left(L_{+}^{p(\cdot)}(\mathbb{R}), L^{p(\cdot)}\left(\mathbb{R}_{+}\right)\right) .
$$

Then,

$$
\begin{aligned}
W_{\phi \varphi} & =W_{\phi} \ell_{0} W_{\varphi}+H_{\phi} \ell_{0} H_{\tilde{\varphi}} \\
H_{\phi \varphi} & =W_{\phi} \ell_{0} H_{\varphi}+H_{\phi} \ell_{0} W_{\widetilde{\varphi}} .
\end{aligned}
$$

In what follows, we will also make use of the identities $J Q=P J, J P=Q J, J^{2}=I, J W_{\phi}^{0} J=W_{\widetilde{\phi}}^{0}$.

\section{ODD ASYMMETRIC FACTORIZATION}

Let us introduce the following operators

$$
P_{J}:=\frac{I+J}{2} \text { and } Q_{J}:=\frac{I-J}{2}
$$

acting on $L^{p(\cdot)}(\mathbb{R})$. Because $J^{2}=I$, these operators are complementary projections. Let us denote the image of $P_{J}$ by $L_{\text {even }}^{p(\cdot)}(\mathbb{R})$ :

$$
L_{\text {even }}^{p(\cdot)}(\mathbb{R}):=\left\{f \in L^{p(\cdot)}(\mathbb{R}): f=\widetilde{f}\right\} .
$$

Additionally, we will also make use of the space $L_{\text {odd }}^{p(\cdot)}(\mathbb{R})$ defined by

$$
L_{\text {odd }}^{p(\cdot)}(\mathbb{R}):=\left\{f \in L^{p(\cdot)}(\mathbb{R}): f=-\widetilde{f}\right\} .
$$


Definition 4 A function $\phi \in G \mathcal{M}_{p(\cdot)}(\mathbb{R})$ is said to admit an odd asymmetric factorization in $L^{p(\cdot)}(\mathbb{R})$, for $p(\cdot) \in$ $\mathcal{B}_{e}(\mathbb{R})$, if it admits a representation

$$
\phi(x)=\phi_{-}(x)\left(\frac{x-i}{x+i}\right)^{k} \phi_{o}(x), \quad x \in \mathbb{R}, k \in \mathbb{Z},
$$

such that:

(i) $\frac{2^{1 / q(\cdot)} x}{(x-i)^{2}} \phi_{-} \in Q L^{p(\cdot)}(\mathbb{R}) \oplus \mathbb{C}, \frac{2^{1 / p(\cdot)}}{(x-i)^{2}} \phi_{-}^{-1} \in Q L^{q(\cdot)}(\mathbb{R}) \oplus \mathbb{C} ;$

(ii) $\frac{2^{1 / p(\cdot)}}{x^{2}+1} \phi_{o} \in L_{\text {odd }}^{q(\cdot)}(\mathbb{R}), \frac{2^{1 / q(\cdot)|x|}}{x^{2}+1} \phi_{o}^{-1} \in L_{o d d}^{p(\cdot)}(\mathbb{R})$;

(iii) the linear operator $V:=P_{J} \mathcal{F}^{-1} \phi_{o}^{-1} \cdot(I-J) P \phi_{-}^{-1} \cdot P: P L^{p(\cdot)}(\mathbb{R}) \rightarrow L_{\text {even }}^{p(\cdot)}(\mathbb{R})$ is bounded.

Proposition 5 Assume that $\phi \in \mathcal{G M}_{p(\cdot)}(\mathbb{R})$, with $p(\cdot) \in \mathcal{B}_{e}(\mathbb{R})$, admits two asymmetric factorizations in $L^{p(\cdot)}(\mathbb{R})$ :

$$
\phi(x)=\phi_{-}^{(1)}(x)\left(\frac{x-i}{x+i}\right)^{k_{1}} \phi_{o}^{(1)}(x)=\phi_{-}^{(2)}(x)\left(\frac{x-i}{x+i}\right)^{k_{2}} \phi_{o}^{(2)}(x), \quad x \in \mathbb{R} .
$$

Then $k_{1}=k_{2}, \phi_{-}^{(1)}=c \phi_{-}^{(2)}$ and $\phi_{o}=c^{-1} \phi_{o}^{(2)}$, for some constant $c \in \mathbb{C} \backslash\{0\}$.

Proof. Let $\phi$ admit two odd asymmetric factorizations in $L^{p(\cdot)}(\mathbb{R})$ :

$$
\phi(x)=\phi_{-}^{(1)}(x)\left(\frac{x-i}{x+i}\right)^{k_{1}} \phi_{o}^{(1)}(x)=\phi_{-}^{(2)}(x)\left(\frac{x-i}{x+i}\right)^{k_{2}} \phi_{o}^{(2)}(x), \quad x \in \mathbb{R}
$$

(where $\phi_{-}^{(1)}, \phi_{-}^{(2)}$ and $\phi_{o}^{(1)}, \phi_{o}^{(2)}$ have the corresponding properties stated in Definition 4). From (8) we immediately have that

$$
\phi_{-}^{(1)}(x)\left(\phi_{-}^{(2)}(x)\right)^{-1}\left(\frac{x-i}{x+i}\right)^{k_{1}-k_{2}}=\phi_{o}^{(2)}(x)\left(\phi_{o}^{(1)}(x)\right)^{-1}, \quad x \in \mathbb{R} .
$$

Assume, without loss of generality, that $k:=k_{1}-k_{2} \leq 0$ and consider the following auxiliary function (where we are denoting by $H_{ \pm}^{1}(\mathbb{R})$ the corresponding Hardy spaces):

$$
\psi(x):=\frac{x}{(x-i)^{4}} \phi_{-}^{(1)}(x)\left(\phi_{-}^{(2)}(x)\right)^{-1} \in H_{-}^{1}(\mathbb{R}) .
$$

A direct computation yields that

$$
\left.\widetilde{\psi}(x):=\frac{-x}{(x+i)^{4}}{\widetilde{\phi_{-}}}^{(1)}(x){\widetilde{\phi_{-}}}^{(2)}(x)\right)^{-1} \in H_{+}^{1}(\mathbb{R}) .
$$

The right-hand side of (9) is an even function (since it is a product of two odd functions). Hence, from (9), we immediately obtain that:

$$
\phi_{-}^{(1)}(x)\left(\phi_{-}^{(2)}(x)\right)^{-1}\left(\frac{x-i}{x+i}\right)^{2 k}=\widetilde{\phi}_{-}^{(1)}(x)\left(\widetilde{\phi}_{-}^{(2)}(x)\right)^{-1} .
$$

This identity together with (10) and (11), lead to the conclusion that

$$
\psi(x)\left(\frac{x-i}{x+i}\right)^{2 k+4}=-\widetilde{\psi}(x) .
$$

Due to the inclusions in (10) and (11), if $2 k+4 \leq 0$, then from (12) we immediately obtain that $\psi$ is identically zero and hence we would have a contradiction. This means that it only remains the possibilities of $k=-1$ and $k=0$. Let us analyze the case where $k=-1$. In this case, (12) is reduced to the form

$$
(x-i)^{2} \psi(x)=-(x+i)^{2} \widetilde{\psi}(x) .
$$

Hence, using (10)-(11), we have a contradiction which shows that $k$ cannot be equal to -1 . Thus the only possibility which is left for $k$ is to be equal to zero. Therefore, in such a case, $k_{1}=k_{2}$. In this case we will have that

$$
\phi_{-}^{(1)}(x)\left(\phi_{-}^{(2)}(x)\right)^{-1}=\widetilde{\phi}_{-}^{(1)}(x)\left(\widetilde{\phi}_{-}^{(2)}(x)\right)^{-1} .
$$

Consequently, $\phi_{-}^{(1)}(x)\left(\phi_{-}^{(2)}(x)\right)^{-1}=c$ for a constant $c \in \mathbb{C} \backslash\{0\}$. Thus $\phi_{-}^{(1)}=c \phi_{-}^{(2)}$ and $\phi_{o}^{(1)}=c^{-1} \phi_{o}^{(2)}$. 


\section{EQUIVALENT OPERATORS AND THEIR CONSEQUENCES}

In order to relate different classes of operators and to transfer certain properties between the operators under study, we will recall some different types of relations between bounded linear operators.

Considering two bounded linear operators $T: X_{1} \rightarrow X_{2}$ and $S: Y_{1} \rightarrow Y_{2}$ acting between Banach spaces, we say that $T$ and $S$ are equivalent if there are two boundedly invertible linear operators, $E: Y_{2} \rightarrow X_{2}$ and $F: X_{1} \rightarrow Y_{1}$, such that

$$
T=E S F .
$$

Another kind of equivalence relation between linear bounded operators is the equivalence after extension (cf. [1]). We say that the operators $T$ and $S$ are equivalent after extension if there exist two Banach spaces, $Z_{1}$ and $Z_{2}$, such that $T \oplus I_{Z_{1}}$ and $S \oplus I_{Z_{2}}$ are equivalent operators, i.e.,

$$
\left[\begin{array}{cc}
T & 0 \\
0 & I_{Z_{1}}
\end{array}\right]=E\left[\begin{array}{cc}
S & 0 \\
0 & I_{Z_{2}}
\end{array}\right] F
$$

for invertible bounded linear operators $E: Y_{2} \times Z_{2} \rightarrow X_{2} \times Z_{1}$ and $F: X_{1} \times Z_{1} \rightarrow Y_{1} \times Z_{2}$, and where $I_{Z_{1}}$ and $I_{Z_{2}}$ are the identity operators on the $Z_{1}$ and $Z_{2}$ spaces, respectively.

If two operators are equivalent or equivalent after extension, then they belong to the same regularity class (cf. [13]). More precisely, one of these operators is one-sided invertible, two-sided invertible, generalized invertible, Fredholm, $n$-normal, $d$-normal or normally solvable if and only if the other operator enjoys the same property.

Let us continue to consider $\phi \in \mathcal{G M}_{p(\cdot)}(\mathbb{R})$ with $p(\cdot) \in \mathcal{B}_{e}(\mathbb{R})$. We will relate the Wiener-Hopf plus Hankel operator with the following operators:

$$
\begin{aligned}
& \vec{W}_{\phi}:=P \phi \cdot \mathcal{F} P_{J}: L_{\text {even }}^{p(\cdot)}(\mathbb{R}) \rightarrow P L^{p(\cdot)}(\mathbb{R}) \\
& \overleftarrow{W_{\phi}}:=Q_{J} \mathcal{F}^{-1} \phi^{-1} \cdot Q: Q L^{p(\cdot)}(\mathbb{R}) \rightarrow L_{\text {odd }}^{p(\cdot)}(\mathbb{R})
\end{aligned}
$$

It is readily seen that $2 \vec{W}_{\phi}=\left.\mathcal{F} \ell_{0}\left(W_{\phi}+H_{\phi}\right)\right|_{L_{\text {even }}^{p(-)}(\mathbb{R})}$. Moreover, in the frame of the domain of the Wiener-Hopf plus Hankel operator we have the following equivalence relation.

Proposition $6 \quad$ Let $\phi \in \mathcal{G M}_{p(\cdot)}(\mathbb{R})$ with $p(\cdot) \in \mathcal{B}_{e}(\mathbb{R})$. The operator

$$
\overrightarrow{W_{\phi}} \in \mathcal{L}\left(L_{\text {even }}^{p(\cdot)}(\mathbb{R}), P L^{p(\cdot)}(\mathbb{R})\right)
$$

is equivalent to the Wiener-Hopf plus Hankel operator $W_{\phi}+H_{\phi} \in \mathcal{L}\left(L_{+}^{p(\cdot)}(\mathbb{R}), L^{p(\cdot)}\left(\mathbb{R}_{+}\right)\right)$.

Proof. Let us consider the operators

$$
\begin{aligned}
& R_{1}:=(I+J) \ell_{0} r_{+}: L_{+}^{p(\cdot)}(\mathbb{R}) \rightarrow L_{\text {even }}^{p(\cdot)}(\mathbb{R}) \\
& R_{2}:=\frac{1}{2} \ell_{0} r_{+}(I+J): L_{\text {even }}^{p(\cdot)}(\mathbb{R}) \rightarrow L_{+}^{p(\cdot)}(\mathbb{R}) .
\end{aligned}
$$

These operators are inverses to one another and a direct computation yields that

$$
\overrightarrow{W_{\phi}}=\mathcal{F} \ell_{0}\left(W_{\phi}+H_{\phi}\right) R_{2}
$$

which shows explicitly the equivalence relation between $\vec{W}_{\phi}$ and $W_{\phi}+H_{\phi}$.

In the next auxiliary result we recall a usefull equivalence after extension relation [1].

Lemma $7 \quad$ Let $Z_{1}$ and $Z_{2}$ be linear spaces, $A: Z_{1} \rightarrow Z_{2}$ be an invertible linear operator, $P_{1}: Z_{1} \rightarrow Z_{1}$ and $P_{2}: Z_{2} \rightarrow Z_{2}$ be linear projections, and $Q_{1}=I-P_{1}$ and $Q_{2}=I-P_{2}$. Then

$$
P_{2} A P_{1}: \operatorname{im} P_{1} \rightarrow \operatorname{im} P_{2}
$$

and

$$
Q_{1} A^{-1} Q_{2}: \operatorname{im} Q_{2} \rightarrow \operatorname{im} Q_{1}
$$

are equivalent after extension operators. 
Proof. Let $A: X \rightarrow Y$ be an invertible operator with inverse $A^{-1}: Y \rightarrow X$. We can rewrite $A^{-1}$ upon its component spaces defined by the above projections, in the following way:

$$
A^{-1}=\left[\begin{array}{cc}
P_{1} A^{-1} Q_{2} & P_{1} A^{-1} P_{2} \\
Q_{1} A^{-1} Q_{2} & Q_{1} A^{-1} P_{2}
\end{array}\right]: Q_{2} Y \times P_{2} Y \rightarrow P_{1} X \times Q_{1} X .
$$

Using this way of writing $A^{-1}$, a direct computation yields

$$
\left[\begin{array}{cc}
Q_{1} A^{-1} Q_{2} & 0 \\
0 & P_{2}
\end{array}\right]=B\left[\begin{array}{cc}
P_{2} A P_{1} & 0 \\
0 & Q_{1}
\end{array}\right] A^{-1},
$$

where $B$ is the invertible matrix operator defined by

$$
B=\left[\begin{array}{cc}
1 & -Q_{1} A^{-1} P_{2} \\
0 & 1
\end{array}\right]\left[\begin{array}{cc}
0 & Q_{1} \\
P_{2} & P_{2} A Q_{1}
\end{array}\right] .
$$

Thus, (15) exhibits precisely an explicit form of an equivalence after extension relation between $Q_{1} A^{-1} Q_{2}$ and $P_{2} A P_{1}$.

Proposition $8 \quad$ Let $\phi \in G \mathcal{M}_{p(\cdot)}(\mathbb{R})$ with $p(\cdot) \in \mathcal{B}_{e}(\mathbb{R})$. The operator

$$
\overrightarrow{W_{\phi}}: L_{\text {even }}^{p(\cdot)}(\mathbb{R}) \rightarrow P L^{p(\cdot)}(\mathbb{R})
$$

is equivalent after extension to

$$
\overleftarrow{W_{\phi}}: Q L^{p(\cdot)}(\mathbb{R}) \rightarrow L_{\text {odd }}^{p(\cdot)}(\mathbb{R})
$$

Proof. The result follows directly from Lemma 7. Indeed, by choosing

$$
P_{1}=P_{J}, \quad P_{2}=P, \quad Q_{1}=Q_{J}, \quad Q_{2}=Q,
$$

we derive from Lemma 7 an explicit equivalence after extension relation between $P \phi \cdot \mathcal{F} P_{J}$ and $Q_{J} \mathcal{F}^{-1} \phi^{-1} \cdot Q$.

The next result is one of the direct consequences of the just presented equivalence and equivalence after extension relations.

Corollary 9 Let $\phi \in \mathcal{G M}_{p(\cdot)}(\mathbb{R})$ with $p(\cdot) \in \mathcal{B}_{e}(\mathbb{R})$. The following assertions are equivalent:

(i) $W_{\phi}+H_{\phi} \in \mathcal{L}\left(L_{+}^{p(\cdot)}(\mathbb{R}), L^{p(\cdot)}\left(\mathbb{R}_{+}\right)\right)$is invertible;

(ii) ${\overrightarrow{W_{\phi}}}_{\boldsymbol{L}} \in \mathcal{L}\left(L_{\text {even }}^{p(\cdot)}(\mathbb{R}), P L^{p(\cdot)}(\mathbb{R})\right)$ is invertible;

(iii) $\overleftarrow{W}_{\phi} \in \mathcal{L}\left(Q L^{p(\cdot)}(\mathbb{R}), L_{\text {odd }}^{p(\cdot)}(\mathbb{R})\right)$ is invertible

Lemma $10 \quad$ Let $\phi \in G \mathcal{M}_{p(\cdot)}(\mathbb{R})$ with $p(\cdot) \in \mathcal{B}_{e}(\mathbb{R})$. Suppose that $\vec{W}_{\phi}$ is invertible. Then there exists functions $f_{-} \neq 0$ and $f_{o}$ such that

$$
f_{-}(x)=\phi(x) f_{o}(x), \quad x \in \mathbb{R},
$$

and

$$
\frac{2^{1 / q(\cdot)} x}{(x-i)^{2}} f_{-} \in Q L^{p(\cdot)}(\mathbb{R}) \oplus \mathbb{C}, \quad \frac{2^{1 / q(\cdot)}|x|}{x^{2}+1} f_{o} \in L_{o d d}^{p(\cdot)}(\mathbb{R})
$$

Proof. If $\vec{W}_{\phi}$ is invertible, then im $\vec{W}_{\phi}=P L^{p(\cdot)}(\mathbb{R})$. Thus, there exists a function $h_{e} \in L_{\text {even }}^{p(\cdot)}(\mathbb{R}) \backslash\{0\}$ such that $\left(\vec{W}_{\phi} h_{e}\right)(x)=(x-i) /(x+i)$ (where we recall that $\left.(x-i) /(x+i) \in P L^{p(\cdot)}(\mathbb{R})\right)$. Defining $\hat{h}_{e}:=\mathcal{F} h_{e}$, we have therefore that $\hat{h}_{e} \in L_{\text {even }}^{p(\cdot)}(\mathbb{R}) \backslash\{0\}$ and

$$
P \phi(x) \hat{h}_{e}(x)-\frac{x-i}{x+i}=0
$$


Thus,

$$
\phi(x) \hat{h}_{e}(x)-\frac{x-i}{x+i}=g_{-}(x), \quad \text { with } g_{-} \in Q L^{p(\cdot)}(\mathbb{R})
$$

and consequently,

$$
\left(\frac{x+i}{x-i}\right) \phi(x) \hat{h}_{e}(x)=\left(\frac{x+i}{x-i}\right) g_{-}(x)+1 .
$$

Let $h_{-}(x)=\left(\frac{x+i}{x-i}\right) g_{-}(x)+1$. It follows that $h_{-} \in Q L^{p(\cdot)}(\mathbb{R}) \oplus \mathbb{C}\left(h_{-} \neq 0\right)$ and $h_{-}(x)=\left(\frac{x+i}{x-i}\right) \phi(x) \hat{h}_{e}(x)$.

If defining $f_{-}(x):=2^{-1 / q(x)} \frac{(x-i)^{2}}{x} h_{-}(x)$, we obtain that

$$
\frac{2^{1 / q(x)} x}{(x-i)^{2}} f_{-}(x)=\left(\frac{x+i}{x-i}\right) \phi(x) \hat{h}_{e}(x) \in Q L^{p(\cdot)}(\mathbb{R}) \oplus \mathbb{C} .
$$

Thus, $f_{-}$satisfies the required condition in (17).

On defining $f_{o}(x):=2^{-1 / q(x)} \frac{x^{2}+1}{x} \hat{h}_{e}(x)$, we have that $\frac{2^{1 / q(x)}|x|}{x^{2}+1} f_{o}(x) \in L_{\text {odd }}^{p(\cdot)}(\mathbb{R})$ and $f_{o}$ is an odd function. Indeed, noticing that the circumstance of $p(\cdot)$ be an even function implies that $q(\cdot)$ is also an even function, it follows

$$
f_{o}(-x)=-2^{-1 / q(x)} \frac{x^{2}+1}{x} \hat{h}_{e}(x)=-f_{o}(x) .
$$

Lemma 11 Let $\phi \in \mathcal{G} \mathcal{M}_{p(\cdot)}(\mathbb{R})$ with $p(\cdot) \in \mathcal{B}_{e}(\mathbb{R})$. Suppose that $\overleftarrow{W_{\phi}}$ is invertible. Then there exist functions $g_{-} \neq 0$ and $g_{o}$ such that

$$
g_{-}(x)=g_{o}(x) \phi^{-1}(x), \quad x \in \mathbb{R},
$$

and

$$
\frac{2^{1 / p(\cdot)}}{(x-i)^{2}} g_{-} \in Q L^{q(\cdot)}(\mathbb{R}) \oplus \mathbb{C}, \quad \frac{2^{1 / p(\cdot)}}{x^{2}+1} g_{o} \in L_{o d d}^{q(\cdot)}(\mathbb{R}) .
$$

Proof. If $\overleftarrow{W_{\phi}} \in \mathcal{L}\left(Q L^{p(\cdot)}(\mathbb{R}), L_{\text {odd }}^{p(\cdot)}(\mathbb{R})\right)$ is invertible, then $\left(\overleftarrow{W_{\phi}}\right)^{*}=Q \overline{\phi^{-1} \cdot \mathcal{F}^{-1}} Q_{J} \in \mathcal{L}\left(L_{\text {odd }}^{q(\cdot)}(\mathbb{R}), Q L^{q(\cdot)}(\mathbb{R})\right)$ is also invertible.

Let $C: \varphi \mapsto \bar{\varphi}$ be the operator of complex conjugation on $L^{q(\cdot)}(\mathbb{R})$. Since $S C+C S=0$ (cf. [18, Lemma 15.1]), we have that

$$
C \overline{\phi^{-1} \cdot \mathcal{F}^{-1}} C=\phi^{-1} \cdot \mathcal{F}^{-1}, \quad C Q C=P .
$$

Consequently,

$$
P \phi^{-1} \cdot \mathcal{F}^{-1} Q_{J}=C\left(Q \overline{\phi^{-1} \cdot \mathcal{F}^{-1}} Q_{J}\right) C
$$

is also invertible and thus, $\operatorname{im}\left(P \phi^{-1} \cdot \mathcal{F}^{-1} Q_{J}\right)=P L^{q(\cdot)}(\mathbb{R})$.

Let $h_{o} \in L_{o d d}^{q(\cdot)}(\mathbb{R}) \backslash\{0\}$ and define $\breve{h}_{o}:=\mathcal{F}^{-1} h_{o}$ such that

$$
P \phi^{-1}(x) \check{h}_{o}(x)=\frac{x-i}{x+i},
$$

i.e.

$$
P\left(\phi^{-1}(x) \check{h}_{o}(x)-\frac{x-i}{x+i}\right)=0 .
$$

It follows that that $\breve{h}_{o} \in L_{\text {odd }}^{q(\cdot)}(\mathbb{R}) \backslash\{0\}$ and

$$
\phi^{-1}(x) \check{h}_{o}(x)-\frac{x-i}{x+i}=f_{-}(x), \quad \text { with } f_{-} \in Q L^{q(\cdot)}(\mathbb{R}) .
$$

Consequently,

$$
\left(\frac{x+i}{x-i}\right) \phi^{-1}(x) \breve{h}_{o}(x)=\left(\frac{x+i}{x-i}\right) f_{-}(x)+1 .
$$


Let

$$
h_{-}(x):=\left(\frac{x+i}{x-i}\right) f_{-}(x)+1
$$

It follows that $h_{-} \in Q L^{q(\cdot)}(\mathbb{R}) \oplus \mathbb{C}$.

If defining $g_{-}(x):=2^{-1 / p(x)}(x-i)^{2} h_{-}(x)(x \neq 0)$, we obtain that $\frac{2^{1 / p(x)}}{(x-i)^{2}} g_{-}(x) \in Q L^{q(\cdot)}(\mathbb{R}) \oplus \mathbb{C}$ and

$$
g_{-}(x)=2^{-1 / p(x)}\left(x^{2}+1\right) \phi^{-1}(x) \check{h}_{o}(x) .
$$

Considering $g_{o}(x):=2^{-1 / p(x)}\left(x^{2}+1\right) \breve{h}_{o}(x)$, we have that $g_{o}$ is an odd function and

$$
\frac{2^{1 / p(\cdot)}}{x^{2}+1} g_{o}(x) \in L_{o d d}^{q(\cdot)}(\mathbb{R})
$$

Thus, we obtain the desired factorization

$$
g_{-}(x)=\phi^{-1}(x) g_{o}(x)
$$

with $g_{-}$and $g_{o}$ satisfying (19).

\section{INVERTIBILITY CRITERION}

We are now in condition to present the main result of this work.

Theorem 12 Let $\phi \in \mathcal{G M}_{p(\cdot)}(\mathbb{R})$ with $p(\cdot) \in \mathcal{B}_{e}(\mathbb{R})$. The Wiener-Hopf plus Hankel operator

$$
W_{\phi}+H_{\phi}: L_{+}^{p(\cdot)}(\mathbb{R}) \rightarrow L^{p(\cdot)}\left(\mathbb{R}_{+}\right)
$$

is invertible if and only if $\phi$ admits an odd asymmetric factorization $\phi=\phi_{-} \phi_{o}$ in $L^{p(\cdot)}(\mathbb{R})$ with a factorization index $k=0$.

Proof. Suppose that $\phi$ admits an odd asymmetric factorization with $k=0$. Let us prove that $\vec{W}_{\phi}$ is invertible. First, notice that we have

$$
\text { ker } \vec{W}_{\phi}=\{0\} \text {. }
$$

Indeed, if $\vec{W}_{\phi} g_{e}=0$, for $g_{e} \in L_{e v e n}^{p(\cdot)}(\mathbb{R})$, then

$$
P \phi_{-} \phi_{o} \mathcal{F} g_{e}=0
$$

and consequently,

$$
\phi_{-} \phi_{o} \mathcal{F} g_{e}=g_{-} \in Q L^{p(\cdot)}(\mathbb{R})
$$

It follows that

$$
\phi_{o} \mathcal{F} g_{e}=\phi_{-}^{-1} g_{-},
$$

where $\phi_{o} \mathcal{F} g_{e}$ is an odd factor which implies that $\phi_{-}^{-1} g_{-}=-\widetilde{\phi_{-}^{-1}} \widetilde{g_{-}}$. Therefore, having in mind the above inclusions, it follows

$$
\frac{2^{1 / p(\cdot)}}{(x-i)^{2}} \phi_{-}^{-1} g_{-} \in H_{-}^{1}(\mathbb{R})
$$

and

$$
\frac{2^{1 / p(\cdot)}}{(x+i)^{2}} \widetilde{\phi_{-}^{-1}} \widetilde{g_{-}} \in H_{+}^{1}(\mathbb{R})
$$

and we conclude that the odd function $\phi_{-}^{-1} g_{-}$is the zero function and, consequently, $\phi_{o} \mathcal{F} g_{e}=0$. Because $\phi_{o} \neq 0$ a.e., it follows that $g_{e}=0$, that is,

$$
\text { ker } \vec{W}_{\phi}=\{0\}
$$

(i.e., $\vec{W}_{\phi}$ is injective). 
Let us now deduce that $\vec{W}_{\phi}$ is surjective. By (iii) of Definition 4 , we have that

$$
V:=P_{J} \mathcal{F}^{-1} \phi_{o}^{-1} \cdot(I-J) P \phi_{-}^{-1} \cdot P: P L^{p(\cdot)}(\mathbb{R}) \rightarrow L_{\text {even }}^{p(\cdot)}(\mathbb{R})
$$

is a bounded linear operator. Let $f \in P L^{p(\cdot)}(\mathbb{R})$. We have

$$
\begin{aligned}
\vec{W}_{\phi} V f & =\left(P \phi_{-} \phi_{o} \cdot \mathcal{F} P_{J} \mathcal{F}^{-1} \phi_{o}^{-1} \cdot(I-J) P \phi_{-}^{-1} \cdot P\right) f \\
& =\frac{1}{2}\left(P \phi_{-} \phi_{o} \phi_{o}^{-1} \cdot(I-J) P \phi_{-}^{-1} \cdot P+P \phi_{-} \phi_{o} \cdot J \phi_{o}^{-1}(I-J) P \phi_{-}^{-1} \cdot P\right) f \\
& =\frac{1}{2}\left(P \phi_{-} \cdot(I-J) P \phi_{-}^{-1} \cdot P-P \phi_{-} \cdot(J-I) P \phi_{-}^{-1} \cdot P\right) f \\
& =\frac{1}{2}\left(P \phi_{-} \cdot P \phi_{-}^{-1} \cdot P-P \phi_{-} \cdot J P \phi_{-}^{-1} \cdot P-P \phi_{-} \cdot J P \phi_{-}^{-1} \cdot P+P \phi_{-} \cdot P \phi_{-}^{-1} \cdot P\right) f \\
& =\left(P \phi_{-} \cdot P \phi_{-}^{-1} \cdot P-P \phi_{-} \cdot J P \phi_{-}^{-1} \cdot P\right) f \\
& =\mathcal{F} \ell_{0}\left(W_{\phi_{-}} \ell_{0} W_{\phi_{-}^{-1}}-H_{\phi_{-}} \ell_{0} W_{\phi_{-}^{-1}}\right) \ell_{0} r_{+} \mathcal{F} f \\
& =P f \\
& =f,
\end{aligned}
$$

by Proposition 3 and using the fact that $H_{\phi_{-}}=0$. Since both $\vec{W}_{\phi}$ and $V$ are bounded, it results that $\vec{W}_{\phi} V=P$. This proves that $\vec{W}_{\phi}$ is surjective .

Thus, if we have an odd asymmetric factorization in $L^{p(\cdot)}(\mathbb{R})$ with $k=0$, then $\vec{W}_{\phi}$ is invertible, and so this is also the case for $W_{\phi}+H_{\phi}$ (cf. Corollary 9).

Suppose now that $W_{\phi}+H_{\phi}$ is invertible (and consequently, $\vec{W}_{\phi}$ and $\overleftarrow{W_{\phi}}$ are also invertible operators). Applying Lemmas 10 and 11, it follows that there exist functions $f_{-}=\phi f_{o}$ and $g_{-}=g_{o} \phi^{-1}$ enjoying the appropriate properties presented in the just mentioned lemmas. Multiplying the corresponding elements in the last two identities, we obtain that

$$
g_{-} f_{-}=g_{o} f_{o}
$$

and it follows that $g_{-} f_{-}=g_{o} f_{o}=: C$ is a nonzero constant.

Now defining $\phi_{-}:=f_{-}=C g_{-}^{-1}$ and $\phi_{o}:=f_{o}^{-1}=g_{o} C^{-1}$ it holds

$$
\phi=\phi_{-} \phi_{o}
$$

It also holds that

$$
V: P L^{p(\cdot)}(\mathbb{R}) \rightarrow L_{\text {even }}^{p(\cdot)}(\mathbb{R})
$$

is a bounded linear operator since, as we have already proved, it is the (right) inverse of $\vec{W}_{\phi}$. Therefore, we have just concluded that $\phi$ admits an even asymmetric factorization in $L^{p(\cdot)}(\mathbb{R})$ with zero index.

\section{ACKNOWLEDGMENTS}

This work was supported in part by FCT-Portuguese Foundation for Science and Technology through the Center for Research and Development in Mathematics and Applications (CIDMA) of Universidade de Aveiro, within project UID/MAT/04106/2013. A. S. Silva is also supported by FCT through the postdoctoral scholarship SFRH/BPD/96763/2013.

\section{REFERENCES}

[1] H. Bart and V. È. Tsekanovskiŭ, "Matricial coupling and equivalence after extension", Oper. Theory Adv. Appl. 59, 143-160 (1992). 
[2] E. Basor and T. Ehrhardt, "Factorization theory for a class of Toeplitz + Hankel operators", J. Operator Theory 51(2), 411-433 (2004).

[3] G. Bogveradze and L. P. Castro, "Wiener-Hopf plus Hankel operators on the real line with unitary and sectorial symbols", Contemporary Mathematics 414, 77-85 (2006).

[4] G. Bogveradze and L. P. Castro, "Invertibility characterization of Wiener-Hopf plus Hankel operators via odd asymmetric factorizations", Banach J. Math. Anal. 3(1), 1-18 (2009).

[5] A. Böttcher, Yu. I. Karlovich and I. M. Spitkovsky, Convolution Operators and Factorization of Almost Periodic Matrix Functions (Birkhäuser, Basel, 2002).

[6] L. P. Castro, "Regularity of convolution type operators with PC symbols in Bessel potential spaces over two finite intervals", Mathematische Nachrichten 261-262, 23-36 (2003).

[7] L. P. Castro and D. Kapanadze, "The impedance boundary-value problem of diffraction by a strip", Journal of Mathematical Analysis and Applications 337, 1031-1040 (2008).

[8] L. P. Castro and D. Kapanadze, "Mixed boundary value problems of diffraction by a half-plane with an obstacle perpendicular to the boundary", Mathematical Methods in Applied Sciences 37, 1412-1427 (2014).

[9] L. P. Castro and A. S. Silva, "Invertibility of matrix Wiener-Hopf plus Hankel operators with symbols producing a positive numerical range", Z. Anal. Anwend. 28(1), 119-127 (2009).

[10] L. P. Castro and A. S. Silva, "Invertibility criteria for Wiener-Hopf plus Hankel operators with different almost periodic Fourier symbol matrices", Annali di Matematica Pura ed Applicata 192, 1141-1152 (2013).

[11] L. P. Castro and A. S. Silva, "Interplay of Wiener-Hopf and Hankel operators with almost periodic Fourier symbols on standard and variable exponent Lebesgue spaces", Annals of Functional Analysis 6(2), 49-59 (2015).

[12] L. P. Castro and F.-O. Speck, "On the characterization of the intermediate space in generalized factorizations", Mathematische Nachrichten 176, 39-54 (1995).

[13] L. P. Castro and F.-O. Speck, "Regularity properties and generalized inverses of delta-related operators", Z. Anal. Anwend. 17, 577 - 598 (1998).

[14] L. P. Castro and F.-O. Speck, "Inversion of matrix convolution type operators with symmetry", Port. Math. 62, 193-216 (2005).

[15] D. Cruz-Uribe, A. Fiorenza, C. J. Neugebauer, "The maximal function on variable $L^{p}$ spaces", Ann. Acad. Sci. Fenn. Math. 28, 223-238 (2003).

[16] L. Diening, P. Harjulehto, P. Hästö and M. Rư̌̌zička, Lebesgue and Sobolev Spaces with Variable Exponents, Lecture Notes in Math. 2017 (Springer, Heidelberg, 2011).

[17] T. Ehrhardt, Factorization Theory for Toeplitz plus Hankel Operators and Singular Integral Operators with Flip (Habilitation Thesis, Technischen Universitität Chemnitz, 2004).

[18] N. Karapetiants and S. Samko, Equations with Involutive Operators (Birkhäuser, Boston, 2001).

[19] A. Yu. Karlovich and I. M. Spitkovsky, "On singular integral operators with semi-almost periodic coefficients on variable Lebesgue spaces", J. Math. Anal. Appl. 384(2), 706-725 (2011).

[20] V. Kokilashvili, A. Meskhi, H. Rafeiro, S. Samko, Integral Operators in Non-Standard Function Spaces. Vol. 1: Variable Exponent Lebesgue and Amalgam Spaces, Operator Theory: Advances and Applications 248 (Birkhäuser, Basel, 2016).

[21] O. Kováčik and J. Rákosník, “On spaces $L^{p(x)}$ and $W^{k, p(x) ”, ~ C z e c h o s l o v a k ~ M a t h . ~ J . ~ 41(4), ~ 592-618 ~(1991) . ~}$

[22] W. Orlicz, "Über konjugierte Exponentenfolgen”, Studia Math. 3, 200-212 (1931).

[23] S. Samko, "Convolution type operators in Lp(x)", Integral Transforms and Special Functions 7, 123-144 (1998). 